\title{
Análisis cualitativo de la reacción al cambio de tres generaciones de empleados en la industria maquiladora
}

\author{
Qualitative analysis of the reaction to the change of three generations of employees \\ in the maquiladora industry
}

\author{
HERNÁNDEZ-PALOMINO, Jorge Arturo*†
}

Instituto Tecnológico de Ciudad Juárez.

ID $1^{\text {er }}$ Autor: Jorge Arturo, Hernández-Palomino / ORC ID: 0000-0001-8372-735X, arXiv Author ID: jorghdez1, CVU CONACYT ID: 177323

DOI: $10.35429 / J T M S .2019 .16 .5 .16 .35$

Recibido 04 de Julio 2019; Aceptado 30 Septiembre, 2019

\section{Resumen}

Este estudio analiza con un diseño cualitativo y un enfoque fenomenológico las reacciones al cambio de tres generaciones de empleados trabajando en la industria maquiladora en Ciudad Juárez. El estudio empírico se llevó mediante la entrevista a 27 empleados en el año 2019. De dicho análisis, surgieron cinco elementos clave en la aceptación o resistencia al cambio organizacional: la percepción generacional, la percepción al cambio, la comunicación, los impactos y las afectaciones Estudios analizando diferentes cohortes generacionales y las reacciones al cambio no se han realizado en México. Los gerentes de las empresas y académicos podrían beneficiarse de los resultados de este estudio y ajustar las estrategias de cambio fundamentados en el comportamiento predicho de los diferentes cohortes generacionales Dado que este estudio se enfoca en empleados de la industria maquiladora solamente, el tamaño de la muestra es pequeño y limitado a una localización geográfica. Adicionalmente, los resultados no pueden ser generalizados a todas las empresas. El método cualitativo de entrevistas limita la posibilidad de generalizar sus resultados. El análisis para futuras investigaciones es presentado.

Cambio organizacional, Cohortes generacionales, Maquiladoras

\begin{abstract}
This study analyzes with a qualitative design and a phenomenological approach the reactions to change of three generations of employees working in the maquiladora industry in Ciudad Juárez. The empirical study was conducted through the interview of 27 employees in 2019. From this analysis, five key elements emerged in the acceptance or resistance to organizational change: generational perception, perception of change, communication, impacts and impacts Studies analyzing different generational cohorts and reactions to change have not been carried out in Mexico. The managers of the companies and academics could benefit from the results of this study and adjust the strategies of change based on the predicted behavior of the different generational cohorts. Given that this study focuses on employees of the maquiladora industry only, the size of the sample is small and limited to a geographical location. Additionally, the results can not be generalized to all companies. The qualitative method of interviews limits the possibility of generalizing its results. The analysis for future research is presented.
\end{abstract}

Organizational change, Generattional cohorts, Maquilas

Citación: HERNÁNDEZ-PALOMINO, Jorge Arturo. Análisis cualitativo de la reacción al cambio de tres generaciones de empleados en la industria maquiladora. Revista Transdisciplinaria de Estudios Migratorios. 2019, 5-16: 16-35

\footnotetext{
* Correspondencia al Autor (correo electrónico: mt00831@ yahoo.com.mx)

$\dagger$ Investigador contribuyendo como primer Autor.
} 


\section{Introducción}

Actualmente, las empresas han desarrollado sus estructuras organizacionales considerando las necesidades del cohorte generacional de los baby boomers. Desafortunadamente, este tipo de integración no se ajusta a la convivencia actual de tres diferentes generaciones de empleados. Los empleados de las generaciones $\mathrm{X}$ y $\mathrm{Y}$ se están volviendo mayoría. Los baby boomers cada vez son menos debido a la cantidad que se están retirando. Este cambio en la composición de la fuerza laboral demanda cambios en el liderazgo, estructura y efectividad organizacional.

De esta manera, las empresas deben realizar ajustes y adaptarse a la evolución de su estructura organizacional demográfica. Para seguir siendo competitivas las empresas deben adaptarse a estos cambios.

En un entorno empresarial cada vez más volátil, las empresas son retadas ante la necesidad de un cambio a un ritmo creciente. De acuerdo con Higgs y Rowland, el cambio organizacional es complejo (2005). Liderar organizaciones a través del cambio representa el mayor desafío del liderazgo (Kotter, 2007).

La resistencia al cambio es una reacción natural que hace que liderar el cambio sea aún más desafiante (Hernández, 2018). A esta complejidad se suman las tres generaciones diferentes de empleados con respuestas y reacciones potencialmente diferentes al cambio organizacional (Lancaster y Stillman, 2002; Jeffries y Hunte, 2003, White, 2006). Actualmente, los líderes saben poco acerca de cómo la respuesta al cambio organizacional varía entre generaciones.

En este estudio se investigó las diferencias en respuesta al cambio organizacional que surgen en función de su pertenencia generacional. Según Coleman, solo después de un cambio importante el $37 \%$ de los empleados estarán comprometidos, el $25 \%$ se resistirá al cambio y el $38 \%$ aceptará el cambio, solo necesitará más apoyo organizacional para que sea más efectivo (2006).

De la misma manera, Axelrod, Axelrod, Jacobs y Beedon (2006) establecieron que la mayor dificultad que enfrentan las empresas es adaptarse y administrar el cambio.
La era de la información ofrece muchas nuevas tecnologías y opciones. Los líderes actuales enfrentan el reto de aprender las opciones tecnológicas, las capacidades que proporcionan las opciones y encontrar la mejor manera de implementar estos cambios tecnológicos (McAfee, 2006).

Establecer las estrategias efectivas de cambio organizacional son un factor importante en el desempeño y competitividad organizacional. Liderar las empresas en forma adecuada a través del cambio es crítico para el éxito de un líder (Murphy, 2005). Administrar diferentes generaciones de empleados es un desafío. Las tres generaciones de los baby boomers, generación $\mathrm{X}$ y generación $\mathrm{Y}$ tienen diferentes experiencias de vida que contribuyen a su visión del mundo y cómo interactúan en las organizaciones (Glass, 2007).

A medida que los tiempos han cambiado, las prácticas de administración y las formas organizativas también han evolucionado. Adicionalmente, las nuevas generaciones de empleados tienen expectativas diferentes Cada una de las generaciones se identifican distintivamente por creencias, valores y normas comunes, y les dan forma los eventos importantes e históricos que dictan la sociedad durante su adolescencia y los años de la juventud adulta (Arsenault, 2004; McNamara, 2005). Estas características generacionales únicas se presentan en las organizaciones, como decisiones y comportamientos que pueden presentar desafíos en el lugar de trabajo (González, 2006).

A medida que las organizaciones continúan experimentando cambios demográficos en la fuerza laboral y en sus niveles de cambio, un problema general que enfrentan los líderes es el poco conocimiento sobre cómo cada una de las generaciones reaccionan de manera única al cambio organizacional. Esta falta de conocimiento puede hacer que fracasen las iniciativas de cambio organizacional. Aunque los estudios retratan el carácter cultural de las generaciones, pocos estudios describen las diferencias entre los miembros de cada cohorte en su respuesta al cambio organizacional. 
Este estudio se encuentra organizado como sigue: la revisión de literatura discute el marco teórico y estudios empíricos previos en las que descansan las hipótesis; posteriormente la metodología es presentada y posteriormente los resultados de la investigación; finalmente, las conclusiones y recomendaciones son abordadas.

\section{Revisión de literatura}

La industria maquiladora se encuentra actualmente bajo la dirección de una compleja mezcla de tres generaciones de gerentes y empleados. Actualmente, las empresas deben buscar la mejor manera de administrar a una fuerza laboral multi-generacional con distintos valores, creando un desafío en la comunicación $y$ el trabajo en equipo.

Las empresas deben considerar los factores psicosociales que pueden adoptar valores por exceso, por defecto o por configuración adversos o desfavorables que pueden afectar negativamente a la salud y seguridad de los trabajadores, así como al desarrollo del trabajo, entendiendo por factores psicosociales aquellas condiciones que se encuentran presentes en una situación laboral y que están directamente relacionadas con la organización, el contenido del trabajo y la realización de la tarea (Juárez, 2007).

Cuando múltiples generaciones trabajan juntas en un departamento o un equipo, pero que no entienden o no conocen los valores generacionales de sus colegas, la producción y competitividad implosiona y salen chispas. Las diferencias generacionales han existido a lo largo del tiempo; sin embargo, el estudio de la historia desde la perspectiva de diferentes generaciones no tenía mucho sentido hasta hace relativamente poco tiempo, debido a la lentitud de la vida y los cambios casi imperceptibles entre una generación y la siguiente (Schlesinger, 1986).

Las diferencias entre generaciones, han llegado a ser más pronunciadas con el surgimiento de la revolución industrial, que nos llevó a la era de la información, y al ritmo vertiginoso en que vivimos hoy en día. El primer teórico en desarrollar sistemáticamente, a principios del siglo $\mathrm{XX}$, el tema de las generaciones como parte de la consciencia histórica y la organización social fue Mannheim (Bengtson, Furlong y Laufer, 1983).
Fue Mannheim quien a través del estudio sociológico de las generaciones proporcionó un medio para expresar que el ritmo del desarrollo histórico tiene sus bases en la ley biológica de la vida útil limitada del hombre y la superposición de las nuevas y viejas generaciones.

En medio de esta concurrencia de varias generaciones en los lugares de trabajo, Lipovetsky (1990) concluye, que vivimos en una era del vacío en la que la sociedad posmoderna individualista sustentada en valores materiales, que no encuentra un fin o metas colectivas, solo un fin individual, lo que no le proporciona ningún tipo de satisfacción. Para el autor, El súper yo se presenta actualmente bajo la forma de imperativos de celebridad, de éxito que, de no realizarse desencadenan una crítica implacable contra el Yo. De este modo se explica la fascinación ejercida por los individuos célebres e ídolos, estimulada por los medios de comunicación, que intensifican los sueños narcisistas de celebridad y de gloria, animan al hombre de la calle a identificarse con las estrellas, a odiar el borreguismo y le hacen aceptar cada vez con más dificultad la banalidad de la existencia cotidiana.

Por ello, las organizaciones tienen que enfrentar lo que Bauman (1991) llama la sociedad líquida. El autor plantea como el ser humano ha transformado la esencia del trabajo como bien común a la esencia del trabajo individual. El trabajador pasa de un trabajo duradero sin vínculos y agradecimiento, a un trabajo inmediato en que los vínculos desaparecen y la gratitud instantánea es lo único que considera.

Lo anterior tiene fundamento en el punto de vista psicosocial tal como lo plantea Peiró (2004), al abordar los cambios recientes en el entorno económico y social. Establece el autor que las transformaciones sociales, económicas y tecnológicas han generado un nuevo panorama en la actividad de las empresas. Dentro de este panorama se puede identificar los movimientos de grupos de personas, donde el cambio de territorio tiene sus propias motivaciones, modalidades, consecuencias, significaciones y sueños (Bustillos, Rincones, Jiménez, Hernández, 2009). 
Lo movimientos migratorios actuales tienen un perfil totalmente diferente al de otros tiempos, por lo que es posible aventurar la idea de una nueva época de las migraciones, por su relevancia y sus implicaciones no solo a nivel local, sino regional y nacional. La salida de trabajadores calificados de diferentes generaciones al extranjero se mueve entre la retorica oficial de falta de reconocimiento y su aplastante realidad (Bustillos, et al, 2009).

La razón de esta salida de trabajadores está relacionada con la búsqueda de mejores empleos con mayores niveles de remuneración, un mayor reconocimiento al trabajo realizado y un medio ambiente más seguro y estable (McKenzie y Sasin, 2007). Al dividir a la gente en generaciones, provoca la objeción obvia desde que los niños nacen y mueren en una línea de tiempo continua, considerando que la categorización de las generaciones es arbitraria (Schlesinger, 1986). Con los años abundan otros problemas, por ejemplo, cada generación es conocida por varios nombres y tiene un inicio divergente que inicia y termina dependiendo del investigador o autor (Markert, 2004).

La confusión persiste, sin embargo, en la obtención de un consenso para la definición de un cohorte generacional, Mannheim (2013) recomienda un período de 30 años para cada generación, un período de tiempo suficiente para que cada generación pueda madurar y aprender lo suficiente para ser influyente. La teoría contemporánea, sin embargo, sostiene que una generación es más o menos cada 20 años, pero todavía hay grandes divergencias en las definiciones.

La fuerza de trabajo de hoy, por primera vez en la historia, se compone de cuatro generaciones (Eisner, 2005), que presenta nuevos retos en las estrategias de organización para atraer, motivar y retener a los empleados (Earle, 2003; Harris, 2005). Hacer frente a estos retos es esencial para mantener la armonía, la productividad y la estabilidad dentro de las organizaciones. El problema, por supuesto, se debe a las diferencias de los valores que dan lugar a las diferentes expectativas de los lugares de trabajo, uno mismo, y los compañeros de trabajo.

Aunque las fechas y nombres de las generaciones difieren para la misma generación, las descripciones de las generaciones son muy consistentes (Eisner, 2005).
Las diferencias en la asignación de rangos de edades o fechas, parece surgir de dos aspectos principales. Uno de ellos es la confusión entre los términos de cohortes y generación, que se utilizan frecuentemente en la literatura de forma indistinta (Markert, 2004). El segundo es el sistema de asignación de grupos generacionales sobre la base de compartir experiencias tempranas sociales y económicas.

Por ello, tres generaciones de los baby boomers, nacidos entre 1946 y 1964; la generación X, nacidos entre 1965 y 1979 y; la generación Y, nacidos después de 1979 al 2000, por lo que también se les conoce como los Millenials han empezado a coincidir en las organizaciones con sus propias necesidades, valores y creencias (Hernández, Espinoza y Aguilar, 2015).

Cada una de las generaciones comparte experiencias comunes en el proceso histórico y social en una etapa similar de la vida que los predispone a una forma común de pensar y a un modo común de comportamiento (Lancaster y Stillman, 2002). El punto es que la combinación de un gran grupo de jóvenes trabajadores entrando y un gran grupo de trabajadores experimentados que están dejando las empresas, forzará a las empresas a encontrar mejores estrategias para continuar utilizando la experiencia y conocimiento de los baby boomers que empiezan a retirarse, retener a los mejores talentos y experiencia de la generación $\mathrm{X} y$ fortalecer a los nuevos integrantes sin experiencia, representados por la generación Y.

\section{Generación de los Baby Boomers}

Esta cohorte generacional nacido entre 1946 y 1964 , crecieron dentro de familias numerosas, lo que da origen al nombre de la generación. El idealismo radical en la década de 1960 se centró en los Baby Boomers en edad universitaria (Howe y Strauss, 2000). Su idealismo los puso en conflicto con el mundo tradicional de la generación de sus padres (Weston, 2006). A pesar de que los integrantes de esta generación representan un gran número potencial de jubilaciones en los próximos 10 a 15 años, no significa necesariamente que los baby boomers se jubilarán por completo. 
Al tiempo que mantiene su idealismo, los baby boomers resultaron ser adictos al trabajo materialistas que eran leales a sus empresas (Tolbize, 2008). Ellos tienden a valorar los títulos de trabajo y buscan la realización personal. De acuerdo con Reynolds (2004), el $31 \%$ de los baby boomers se está moviendo a nuevas líneas de trabajo y el $10 \%$ de ellos ha empezado a trabajar en servicios personales como consultores, asesores financieros y de negocios.

Otra característica importante del grupo generacional de los Baby Boomers es que crecieron en un tiempo anterior al actual auge de la innovación tecnológica. Como resultado de ello, los Baby Boomers están en el extremo tardío de la curva de adopción de tecnología (Simons, 2010). Esta brecha tecnológica pone frecuentemente a los baby boomers en conflicto con las generaciones más jóvenes. A pesar de ser altamente respetuosos de la autoridad, son propensos a resentir el autoritarismo o la pereza y tienden realizar sus gestiones basados en el tiempo (Morton, 2001). Entre las fortalezas que identifican a este grupo es su estilo participativo, y su capacidad para liderar desde el corazón. Sus retos suelen encontrarse en desarrollar sus habilidades de liderazgo, enfrentar los conflictos practicando lo que predican.

\section{Generación X}

El nombre de la Generación X fue acuñado por Coupland (1991), en el título de una novela que describe a esta nueva generación con diferentes valores y las aspiraciones de los Baby Boomers. Mientras que para muchos miembros de la generación no fue de mucho agrado el nombre, la identificación sigue siendo el título más popular para el grupo (Rainer y Rainer, 2011). La generación $X$ también se conoce como la generación Baby-Buster debido a su pequeña población en relación con los Baby Boomers. La generación $X$ luchó para definirse a sí mismos y la literatura refleja el amplio rango de creencias sobre este grupo generacional.

La generación X, los nacidos entre 1965 y 1980 , representan el $34 \%$ de la fuerza de trabajo. A diferencia de otras generaciones de la fuerza de trabajo hoy en día, la Generación X ha recibido una caracterización un tanto negativa, tanto en los medios de comunicación y la literatura (Morton, 2003).
Descripciones tales como perezosos, cínicos, y quejosos (Williams y Nussbaum, 2001) se han incluido con descriptores más positivos tales como inteligentes, independientes, pragmáticos y autosuficientes (Morton, 2003); individualistas, enérgicos, ambiciosos emprendedores, amantes de la libertad y autosuficientes (Eisner, 2005).

La generación $X$ crece ya en un mundo en línea las 24 horas, los siete días a la semana con el teléfono celular, el cable e Internet y viviendo en hogares con altas tasas de divorcios. Ellos no tienen héroes. Un tercio de la generación $X$ creció en la pobreza causada por la crisis económica, el desempleo y las altas tasas de divorcio (Morton, 2003). La generación X se crió en una época en que las necesidades de los niños fueron puestas al final y sobrevivieron la década de 1970 con agitaciones económicas. El aspecto positivo de la generación $X$ es que crecieron en un mundo relativamente pacifico, auge tecnológico y disfrutando de los beneficios de las causas que sus padres iniciaron, como la de los Derechos Civiles, los movimientos de las mujeres y la protección al medio ambiente (Eisner, 2005).

Los miembros de la generación $\mathrm{X}$ no ven el éxito de la misma manera que sus padres. Se caracterizan por ser un grupo escéptico y su desilusión por los valores de sus padres. En el marco de la generación $X$, se identifica el surgimiento de una subcategoría, la de los yuppies. Appelbaum, Serena y Shapiro (2004) identifican a este segmento por un alto poder adquisitivo y una autentica pasión por lucrar social, profesional y económicamente. A finales de la década de los ochenta, el término yuppie, se empezó a utilizar con ciertas características negativas. Esto dio como resultado el agotamiento del modelo y el estilo de vida que proclamaba un todo se vale con la intención de alcanzar el éxito económico y social.

Adicionalmente, podemos identificar dentro de este grupo de los yuppies a los dinkies, vocablo que surge de las palabras en inglés "Double-Income Not Kids Yet", (Doble ingreso, hijos todavía no). Los dinkies son parejas de yuppies que posponen la formación de una familia para dedicarse exclusivamente a sus carreras profesionales. 
Suelen ser profesionales de alto nivel y su éxito lo miden con el mantenimiento de un alto nivel socioeconómico, lo que ha generado críticas por su actitud egoísta y hedonista. Sus valores se caracterizan por el consumismo, por encima de los valores familiares.

Esta generación piensa globalmente, tecnológicamente preparada, divertida, informal y pragmática (Zemke, 2001). Ellos tienden a mantener un equilibrio entre vida y trabajo. Se les reconoce por la frase, trabajar para vivir, no vivir para trabajar (Sturges y Guest, 2004). Son individualistas y ambiciosos y en 1990 su espíritu emprendedor los convirtió en los superestrellas del punto-com. Los miembros de la generación $\mathrm{X}$ miden el éxito a través de trabajos flexibles que les permita equilibrar el trabajo y la vida, para mantener relaciones sanas y estables (Morton, 2003).

\section{Generación Y}

La generación Y, nacida entre 1981 y el 2000 representa el $20 \%$ de la fuerza laboral y por si sola será la generación que llene el vacio que vayan dejando los baby boomers que se vayan retirando. $\mathrm{Si}$ la generación $\mathrm{X}$ puede ser considerada como la generación pérdida, viviendo a la sombra de los baby boomers, la generación $\mathrm{Y}$ puede ser considerada la generación encontrada, quienes fueron adorados por sus padres de la generación de los baby boomers y la generación $\mathrm{X}$ y altamente apreciados por sus abuelos de la generación de los baby boomers y tradicionalistas (Zemke, 2001).

La generación $\mathrm{Y}$, es también conocida como los Milenians, la generación Einstein y es el grupo que está impactando la fuerza de trabajo de hoy y que seguirá impactando por décadas. Los más viejos de ésta generación están a punto de alcanzar los treinta y cinco años. Los miembros de la generación Y son niños deseados $\mathrm{y}$ protegidos por una sociedad que se ha preocupado por su completa seguridad.

Sus miembros son alegres, seguros de sí mismos y enérgicos. Es la generación del Internet, la generación de las tecnologías que cambian constantemente. La generación Y está resultando compleja de integrar en las empresas, considerando sus nuevas expectativas y también se les conoce como la generación excluida. Seguramente serán hijos únicos o tendrán pocos hermanos y su madre también trabaje.
La tecnología no es un problema para ellos, el $91.6 \%$ de los jóvenes entre 16 y 24 años son usuarios de internet. Ese porcentaje desciende al $63.4 \%$ en usuarios entre 35 a 44 años. Al igual que en la generación $X$, la generación Y posee una subcategoría que es identificada como los Cosmopolitan Business People, en español, Personas Cosmopolitas de Negocios. Por las características de la generación a ellos les gusta que los identifiquen en inglés. Ellos representan una élite urbana, la cual cristaliza los valores y estilos de vida de la generación. Se comunican en varios idiomas, principalmente en inglés, son poliglotas, bilingües o trilingües. Poseen niveles de educación altos con posgrados o estudian en universidades de prestigio. Son solteros o casados con pocos hijos y comúnmente su pareja también pertenece a la élite de los Cosmopolitan, lo que genera familias inestables. Buscan carreras brillantes, altos sueldos y adoran las empresas multinacionales.

A este respecto, señala Bauman (2003) que vivimos el fin de la sociedad sólida $\mathrm{y}$ pasamos a la sociedad líquida. La incertidumbre en que vivimos se corresponde a transformaciones como el debilitamiento de los sistemas de seguridad que protegían al individuo y la renuncia a la planificación de largo plazo: el olvido y el desarraigo afectivo se presentan como condición del éxito. Esta nueva sensibilidad exige a los individuos flexibilidad, fragmentación y compartimentación de intereses y afectos, se debe estar siempre bien dispuesto a cambiar de tácticas, a abandonar compromisos y lealtades.

La generación $\mathrm{Y}$ se caracteriza por un nuevo modelo de familia: mascotas y amigos. Sus estilos de vida van se caracteriza por tratar de vivir cerca de sus amigos, pero no juntos. Este estilo de convivencia, les permite crear comunidades sin la necesidad de establecer relaciones formales o de largo plazo. Existen opiniones contradictorias sobre la generación Y (Hoover, 2009; Rawlins, Indvik, y Johnson, 2008; Twenge, Konrath, Foster, Campbell y Bushman, 2008). Los temas comunes entre los estudiosos, que examinaron la generación Y señalaron que las diferencias en la nueva generación incluyen baja aptitud mecánica y una mayor necesidad de apoyo y aliento (FerriReed, 2010). Por otra parte, la opinión general sobre la generación Y es vista como un mito. 
Tienen un aire entre irreverente e ingenuo que no llegamos a descifrar. Nos hablan de igual a igual, no conciben la idea de pagar derecho de piso, eso del sacrificio no les va, los largos horarios les parecen descabellados, quieren hacer sólo lo que les divierte, quieren viajar, no tienen compromiso con la empresa y viven conectados a algo, sea la web, el iPod, el celular, Facebook, y muchas veces todo a la vez. Para ellos, la familia es hoy en día un lugar muy importante. A los jóvenes les gusta estar juntos, les encantan las relaciones en armonía y necesitan, por tanto, un hogar en el que sean sinceras e importantísimas, y en el que la amistad y la familia jueguen un papel esencial.

En definitiva, una familia en la que se pueda compartir actividades con los demás, se pueda pasar el rato y sea posible jugar a algo durante un buen tiempo es pequeña, acogedora e íntima, y ofrece un espacio de protección, seguridad y confianza en un mundo cada vez más grande. Adicionalmente, los miembros de la generación Y no prefieren tener hijos. Ellos han cambiado su preferencia por las mascotas. La popularidad de esta forma de vida ha generado incluso varios nombres, dentro de los cuales uno muy popular es la de los perrhijos y gathijos.

Si bien los miembros de la generación Y valoran trabajar desde casa, al tener mascotas les ha generado problemas de concentración y atención a su trabajo. Esto se ha convertido en otro de los componentes, del por qué ellos no conocen un sueño profundo.

Como resultado de su visión del mundo, la generación Y convive en el mundo laboral con diferentes ideas sobre el orden, las reglas y el liderazgo. Las experiencias de vida de la generación $\mathrm{Y}$, ha creado una generación que posee rasgos tales como la falta de confianza en las corporaciones, un enfoque en el éxito personal, y una perspectiva de carrera a corto plazo (Swenson, 2008). La Generación Y considera muy importante a la flexibilidad laboral y a la conciliación entre la esfera profesional y personal.

Ellos esperan oportunidades de aprendizaje y desarrollo en el trabajo, acceso a una comunicación abierta y fluida y que se respete su estilo de vida. Creen más en la colaboración y la toma conjunta de decisiones que en la jerarquía. Son unos firmes defensores de la responsabilidad individual y quieren libertad para tomar decisiones.
Por otra parte, representan la diversidad, y también la valoran. Son personas emprendedoras y con una gran capacidad para resolver problemas. Por eso valoran positivamente los entornos laborales que impulsan y premian la iniciativa.

Buscan un entorno de trabajo agradable, que fomente las relaciones sociales. No les asusta la rotación laboral y les motiva escalar posiciones, no porque represente más rango o poder, sino porque implica obtener reconocimiento y mayor capacidad para poner en marcha sus iniciativas. La generación Y entra a un ambiente de trabajo conformado por tradiciones y comportamientos moldeados por los Baby Boomers, los tradicionalistas y la Generación X. Estos integrantes usan la tecnología en aspectos muy cercanos de sus vidas. La usan para trabajar, para jugar o simplemente para pasar el tiempo.

El celular siempre está a su lado, incluso al ir a dormir, lo que les garantiza que ellos estarán comunicados todo el tiempo. La generación $\mathrm{Y}$ presenció los ataques a las torres gemelas en Estados Unidos, conocido como el 9/11. Esta generación se distingue por una actitud desafiante y retadora. Esta fue la última generación que vio la Guerra fría por los pocos recuerdos que tiene desde la niñez, en tecnología le toco vivir el auge de Internet, la transición del DOS al Windows, jugó desde el Atari hasta el wii.

Los miembros de la generación $\mathrm{Y}$ crecieron siendo el centro de atención de familias ocupadas con vidas sobre planeadas. En un mundo comunicado las 24 horas, los siete días de la semana con tecnología digital, la generación $\mathrm{Y}$ ha visto más a una edad menor que cualquiera de otra generación (Sujansky, 2004). Son usuarios consumados de la tecnología y creció con internet. Acostumbrados a estar conectados con amigos y familiares en todo momento, los miembros de la generación Y son hijos de padres muy involucrados que los valoran $\mathrm{y}$ ha reducido la brecha generacional entre ellos. Los valores y las actitudes de la generación Y son la lealtad, la inclusión y la autosuficiencia (Eisner, 2005). Es la generación más conocedora de la tecnología y como tal son más abiertos al trabajo en equipo que cualquiera de las generaciones anteriores y se desarrollan en la creación de redes e intercambio de información. 
La generación Y mide el éxito a través de la realización personal en el trabajo, una vida activa fuera del trabajo y su participación en la comunidad. Es la generación mejor educada y si se le agrega su sofisticación tecnológica, las expectativas positivas y su aparente inclinación colectiva, es probable que tengan la fórmula para la grandeza (Zemke, 2001).

\section{La Industria maquiladora en Ciudad Juárez}

Debido a los estragos generados por la Segunda Guerra Mundial, Estados Unidos se vió en la necesidad de contratar trabajadores para trabajar fundamentalmente en el campo y algunas actividades industriales. De esta manera, en 1942 se firmó el acuerdo denominado "Programa Bracero" entre los gobiernos de México y Estados Unidos para establecer la contratación legal de trabajadores mexicanos. En el año inicial se empezó con la contratación de 4,203 trabajadores, para 1951 el número se había elevado a 192,000, la contratación pico se dio en 1956 con 445,000 y para terminar en 1964 con 177,000 .

En total se contrataron aproximadamente 4.6 millones de trabajadores según cifras de las autoridades estadounidenses. Durante la vigencia del Programa Bracero, muchos trabajadores instalaron su residencia en Ciudad Juárez; sin embargo, al finalizar el acuerdo en 1964, esta ciudad se convirtió en el puerto de regreso de más de cuatro millones de mexicanos que habían migrado a los Estados Unidos con permisos de trabajo.

Originalmente, el programa de maquiladoras fue un instrumento para crear empleos. El gobierno mexicano buscaba enfrentar una situación de desempleo grave en la zona fronteriza con una política de excepción, con alcances temporales y regionales delimitados. En cuanto a su temporalidad, se pretendía que en el mediano plazo las plantas de ensamble crearan eslabonamientos productivos, y promovieran una industrialización de base nacional; en cuanto a su alcance regional, el modelo respondía de forma exclusiva a las condiciones de la frontera norte, pues en el resto del país la política industrial continuaba centrada en la protección de la industria mexicana, mediante instrumentos fiscales y arancelarios (Contreras y Munguía, 2007).
Entre las consecuencias del hecho de que el programa de maquiladoras estuviera dirigido a una región específica, pero sobre todo el de tratarse de una política en franco antagonismo con el pensamiento proteccionista dominante en el gobierno federal mexicano, es que en realidad no se diseñó ningún instrumento concreto para promover el objetivo explícito de crear eslabonamientos productivos a través de ellas. En el discurso oficial, las maquiladoras nunca dejaron de considerarse un "mal temporal"; en los hechos, el enfoque gubernamental se limitó a permitir su instalación para combatir el desempleo y generar divisas.

Según tal enfoque, se elaboraron todos los instrumentos que regularon sus actividades durante los primeros 20 años de operación: en 1965 se emitieron las reglas que permitieron la importación temporal de maquinaria e insumos a la franja fronteriza. Como parte del programa industrial fronterizo se creó la política de fomento a la industria maquiladora de exportación, por parte del gobierno federal de Gustavo Díaz Ordaz. Dicha política planteaba la búsqueda de generación de empleos y fortalecer la balanza comercial. La política permitía la entrada de empresas con capital totalmente extranjero, contrario a lo que se permitía a otras empresas de manufactura, hasta el $49 \%$ de inversión extranjera. Adicionalmente, exentaba de impuestos la importación y exportación de insumos y componentes.

Fue hasta 1969 que se creó el parque industrial Antonio J. Bermúdez, en el cual se inició la compañía productora de artículos electrónicos RCA, televisiones; al año siguiente llegaron Convertors, artículos médicos y Essex International de Chihuahua, arneses para Chrysler. Para 1970, tanto las maquiladoras tradicionales como las dedicadas a la tecnología, en el sector automotriz, médico y otros, llegaron hasta las 22 plantas localizadas en esta ciudad.

En 1971 un reglamento nuevo autorizó el establecimiento de plantas en regiones no fronterizas, y la creación de empresas de capital extranjero en su totalidad y en 1977 se definieron los mecanismos de coordinación, para el cumplimiento de las normas aplicables a este tipo de empresas. Todo inició con un puñado de fábricas, que llegaron a sacar a Juárez del desempleo. 
La RCA, Nielsen, Convertors y Essex International, son los algunos de los nombres de empresas que se han difuminado en la mente colectiva de los juarenses con el pasar del tiempo, pero estas plantas manufactureras fueron las que construyeron los cimientos de uno de los modelos industriales más importantes de México.

A 54 años de la llegada de la maquiladora a Ciudad Juárez con el Programa de Industrialización Fronteriza (PIF), la urbe se ha convertido en un punto de referencia de la inversión extranjera, gracias a la confianza que la mano de obra de la región ha desarrollado progresivamente. Los recuerdos de las primeras firmas nacionales y extranjeras -de las que poco o nada queda- aún persisten en la memoria de los gestores de la industria en esta ciudad, sobre todo, los resultados y los cambios sociales aún perduran. En todo ese periodo, los cambios legales fueron precisando las normas administrativas y los mecanismos de operación, pero siempre considerando a las maquiladoras como industrias transitorias, en una situación de excepción (Contreras 2000).

Ciudad Juárez, Chihuahua, México es la principal fuente de empleo de la industria maquiladora del país. A nivel local, la población ocupada se encuentra localizada principalmente en los subsectores de empleo industrial manufacturero (48.4\%), seguido por empleo en áreas comerciales (14.6\%) y de servicios (11.4\%) (INEGI, 2017). De hecho, 1 de cada 10 empleos dentro de ésta industria maquiladora nacional, se generan en dicha localidad. Juárez cuenta con varias empresas multinacionales y en las que su origen de capital se encuentra en Estados Unidos, Canadá, China, Francia, Japón, Corea del Sur, Alemania, entre otros países. De esta manera, los trabajadores mexicanos conviven diariamente con personas de diferentes países y se comunican en diferentes idiomas, confrontando sus raíces sociales y culturales.

La industria maquiladora en México, emplea a más de 2 millones 373,000 personas. Ciudad Juárez, emplea al 12\% del total nacional. Además de ser la ciudad con más personal maquilador del país, Ciudad Juárez tiene las plantas industriales más grandes. De acuerdo con el Instituto Nacional de Estadística y Geografía (Inegi), la media es de 781 personas empleadas por planta, mientras el promedio nacional es de 474 personas por fábrica.
Adicionalmente, la estadística Integral del Programa de la Industria Manufacturera, Maquiladora y de Servicios de Exportación del Inegi, muestra que al mes de febrero del 2018 en Ciudad Juárez existían 322 plantas en las que trabajaban 250 mil 577 empleados.

Sólo en el último año, el número de personas ocupadas por maquiladora en Juárez ha crecido un 8.8 por ciento, muy por encima de la media nacional que es de 4.9 por ciento. Otra característica adicional de Ciudad Juárez, es que más que sufrir la fuga de talentos, se caracteriza por recibir una serie de talentos de los diferentes países de origen o con operaciones de las empresas maquiladoras. Por ello, en México, la salida de trabajadores calificados de diferentes generaciones al extranjero se mueve entre la retorica oficial de falta de reconocimiento y su aplastante realidad (Bustillos, Rincones, Jiménez, Hernández, 2009).

La empresa maquiladora de Ciudad Juárez se ha vuelto un semillero de gente talentosa y bien pagada que abarca a cuatro generaciones de empleados en el mismo lugar de trabajo y que en lo general no aspira al famoso sueño americano (Bustillos, et al 2009). Esto es consistente con Santos (2006), de que las creencias o teorías no son un reflejo del mundo actual, sino que lo que requerimos es una ecología de saberes.

Para administrar de manera efectiva en este entorno, las empresas maquiladoras deben generar esa ecología de saberes (Santos, 2006). Esto es, aprender desaprendiendo, crear políticas flexibles y procedimientos que se adapten a todas las generaciones, algo que no ha sido el estándar para las organizaciones de hoy. La ecología de saberes tiene como base la idea de que el conocimiento es inter-conocimiento. Las nuevas formas de organizar el trabajo buscan una mayor eficiencia y eficacia, una mayor flexibilidad, una optimización de los recursos tecnológicos y una mayor productividad y calidad como diferencia competitiva de la empresa. Todos estos planteamientos tienen fuertes implicaciones sobre las actividades laborales y la cualificación de los trabajadores. Determinados conocimientos, habilidades y destrezas que eran importantes en el desempeño del trabajo resultan ahora prácticamente irrelevantes y competencias que antes ni siquiera se tomaban en consideración resultan, ahora imprescindibles (Peiró, 2004). 
Las empresas de manufactura se integran fundamentalmente de tres generaciones de empleados trabajando juntos. Todos los sistemas cambian de alguna manera ya que reaccionan a los estímulos del medio ambiente en el que operan. Los investigadores en este campo tratan de comprender las formas en que las organizaciones interactúan con ciertos tipos de estímulos que se encuentran frecuentemente en un ambiente organizacional basada en el mercado (Rummler y Brache, 1995).

Este estudio considera la afirmación de los investigadores, que la organización es un sistema complejo que reacciona a los cambios ambientales que pueden venir desde el exterior en términos de los cambios del mercado, la tecnología, o la demografía, o los cambios que puede provenir de componentes internos. Todos estos factores son entradas interdependientes en el sistema organizacional.

La revisión de literatura proporciona los fundamentos de discusión de este estudio. El propósito de este estudio fenomenológico es explorar si existen diferencias o no entre los miembros de la generación de los baby boomers, generación $\mathrm{X}$ y generación $\mathrm{Y}$ en respuesta al cambio organizacional. La revisión de literatura permite establecer que cada generación posee diferentes valores y creencias. Por tanto, los dirigentes de las empresas necesitan conocer estas diferencias. A pesar de la información y las creencias son fundamentales en la toma de decisiones, los valores son el elemento determinante. Los valores dirigen los juicios individuales y dan forma al actuar de una persona.

El propósito fundamental de este estudio fue investigar y describir las experiencias de cada cohorte generacional al cambio. Ser consciente de las características generacionales proporciona inicialmente, un mejor entendimiento de la fuerza laboral y de los motivadores personales de los empleados (Jeffries y Huntle, 2003). Esto es significativo para determinar si los miembros de las tres generaciones de este estudio, los baby boomers, la generación X y la generación Y reaccionan de forma diferente al cambio organizacional.
Si los resultados muestran que existen diferencias en la forma en que cada generación reacciona al cambio, los dirigentes de las empresas podrán tomar en consideración las diferencias generacionales al desarrollar e implantar estrategias que le permita a los empleados adaptarse al cambio.

\section{Metodología}

El estudio actual utilizó un método fenomenológico cualitativo. Un método fenomenológico se ajusta mejor al estudio actual porque la fenomenología describió el significado de la experiencia del individuo con el tema en estudio (Creswell, 2003). Un estudio fenomenológico comunica cómo los individuos perciben, describen, sienten, juzgan, recuerdan y dan sentido a las situaciones (Patton, 2002).

Denzin y Lincoln (1998) establecen que la investigación cualitativa fortalece "la naturaleza social de la realidad y las limitaciones situacionales que son parte de la investigación". Los participantes fueron seleccionados basados en el criterio de la muestra útil. De acuerdo a Creswell (1998), el criterio de la muestra útil es adecuado cuando todos los participantes estudiados representan gente que ha experimentado el fenómeno.

El enfoque cualitativo resultó el más adecuado para responder la pregunta de investigación de si existen o no diferencias generacionales entre los baby boomers, la generación $\mathrm{X}$ y la generación $\mathrm{Y}$ en su respuesta al cambio organizacional. La investigación cualitativa se basa en las opiniones de los participantes, utiliza preguntas amplias y reúne información hecha principalmente de texto. El investigador analiza el texto para formar temas; una El análisis cualitativo es subjetivo y contiene sesgo.

Para mitigar el sesgo, el análisis de datos incluyó la reflexividad donde se reflejó el sesgo como parte del informe (Moustakas, 1994). Para seguir siendo objetivos, los investigadores cualitativos deben estar al tanto de las opiniones personales y ser sinceros acerca de suposiciones previas (Neuman, 2003). Varias razones influyeron para seleccionar un enfoque cualitativo para este estudio. Primero, un método cualitativo enfoca la atención en la importancia de los individuos dentro de la organización (MacDonald, 2007). 
Un enfoque cualitativo es adecuado en estudios exploratorios donde existe poco conocimiento, ya que los procedimientos cualitativos pueden proporcionar información rápida para ayudar a tomar decisiones cuando el tiempo es una preocupación (MacDonald, 2007). El estudio cualitativo proporcionó ricas descripciones de las experiencias vividas de los participantes del estudio.

Un enfoque cualitativo fue el más adecuado para explorar una pregunta sin ideas predeterminadas sobre lo que puede ser descubierto. El enfoque cualitativo proporcionó una exploración con una visión amplia. Los defensores de la investigación cuantitativa pueden cuestionar el mérito de un enfoque cualitativo. La investigación cuantitativa es valiosa para describir tendencias entre variables. Por el contrario, un enfoque cualitativo es mejor para las preguntas de estudio en las que el investigador no conoce las variables (Creswell, 2005). La investigación cualitativa utiliza un enfoque más abierto y profundo que la investigación cuantitativa.

Dado el propósito del estudio, el alcance de la investigación fue un estudio descriptivo fenomenológico. La recolección de la información consistió en una profunda entrevista cualitativa y un cuestionario demográfico de sus características personales. De acuerdo con Moustakas (1994) el uso del modelo fenomenológico permite obtener descripciones comprensivas que proporcionan las bases de un análisis estructural reflexivo que retrata los aspectos fundamentales de la experiencia. Utilizando este método, los datos pueden ser descritos en dos niveles:

Primero, las respuestas de los participantes pueden ser obtenidas a través de preguntas abiertas y diálogo.

Segundo, el investigador describe las estructuras de las experiencias basado en los relatos de los participantes.

El uso del diseño fenomenológico de investigación fue apropiado para este tipo de estudio que requiere de una exploración rigurosa de un tópico poco estudiado en México. Un alcance fenomenológico permite al investigador direccionar detalles en términos de matices y significados (Creswell, 1998). La investigación actual utilizó entrevistas de grupos focales para abordar las preguntas de investigación.
Según Neuman (2003), las entrevistas en persona tienen las tasas de respuesta más altas y permiten una encuesta más larga. Además, las entrevistas cara a cara permiten al investigador observar al participante y reconocer respuestas no verbales. Los grupos focales en el estudio actual fueron grabados en audio y transcritos. Los grupos focales son adecuados cuando se espera que la comunicación entre los participantes proporcione la mejor información y cuando los participantes compartan puntos en común y cooperen entre sí (Creswell, 2005).

Neuman (2003) sugirió que un investigador bien entrenado puede hacer preguntas complejas y puede usar sondeos. En un grupo focal, típicamente de 6 a 12 participantes están en una sala con el investigador para discutir algunos temas (Neuman, 2003). El estudio actual utilizó ocho participantes por grupo focal de cada una de las tres generaciones.

Las entrevistas de grupos focales tienen ventajas y limitaciones (Neuman, 2003). De acuerdo con este autor, una ventaja de las entrevistas de grupos focales es que en un grupo focal los participantes pueden articular libremente opiniones e ideas. Los participantes a menudo se sienten empoderados y pueden interactuar entre sí para explicar sus respuestas. Los investigadores reciben información valiosa sobre cómo los participantes discuten el tema de investigación.

Las limitaciones de los grupos focales incluyen la posibilidad de que las opiniones se vuelvan más intensas después de la discusión grupal. Un grupo focal puede discutir solo un número limitado de temas por sesión. El moderador del grupo focal puede suprimir inadvertidamente la discusión abierta de los participantes. Los investigadores pueden no producir detalles adecuados en el informe (Neuman, 2003).

Las preguntas de investigación para el estudio fenomenológico actual fueron diseñadas para descubrir el fenómeno de la respuesta generacional al cambio organizacional. Las principales preguntas de investigación incluyeron:

1. ¿Qué diferencias existen entre los Baby Boomers, la Generación $X$ y la Generación $\mathrm{Y}$ en respuesta al cambio organizacional?

HERNÁNDEZ-PALOMINO, Jorge Arturo. Análisis cualitativo de la reacción al cambio de tres generaciones de empleados en la industria maquiladora. Revista Transdisciplinaria de Estudios Migratorios. 2019 
2. ¿Cómo pueden los líderes guiar a los miembros de diferentes generaciones a través de iniciativas de cambio?

3. ¿Cómo ven los empleados los comportamientos de cambio de los miembros de diferentes generaciones?

4. ¿Cómo describen los empleados el impacto del cambio en el desempeño organizacional y personal?

5. ¿Qué pueden hacer los líderes para aumentar la aceptación de los empleados de las iniciativas de cambio?

Durante la entrevista, los sondeos exploraron caminos adicionales para investigar la esencia del fenómeno de la respuesta generacional al cambio organizacional.

Este estudio se llevó a cabo durante los meses de enero a junio, del año 2019. La población en este estudio fue los empleados de la industria maquiladora en Ciudad Juárez, Chihuahua, México, con experiencia directa con el cambio organizacional, en niveles de supervisión y gerenciales teniendo a su cargo al menos 20 trabajadores en línea vertical y horizontal. El criterio para elegir a los participantes incluyó el ofrecimiento para ser voluntario y participar en el estudio, la experiencia vivida con el cambio organizacional y la voluntad de compartir experiencias y tiempo para explorar el tema de la respuesta al cambio organizacional.

Según Crow, Wiles, Heath y Charles (2006), el consentimiento informado requiere que el investigador proporcione a los participantes de la investigación información esencial sobre el estudio. Además, el consentimiento informado requiere que los participantes acuerden libremente y tengan el derecho de negarse a participar en el estudio o retirarse sin consecuencias negativas. Se tomaron las medidas necesarias para proporcionar a los participantes del estudio una revelación completa de la intención y el propósito del estudio.

Se tomaron medidas esenciales para cumplir con los estándares de investigación ética. Una preocupación común de investigación ética es el engaño y el encubrimiento. La participación en el estudio fue voluntaria y se informó a los participantes el propósito y la intención de la investigación.
Se informó a los participantes que los resultados del estudio se publicarían, de su derecho a negarse a participar en el estudio y de su derecho a abandonar la entrevista en cualquier momento (Cone y Foster, 2004).

A los participantes se les pidió permiso para grabar en audio las entrevistas. Los participantes firmaron una carta de consentimiento y reconocer su voluntad de participar en la investigación. La carta de consentimiento también informa a los participantes de la información que deben proporcionar y les dice a los participantes qué deben hacer (Cone \& Foster, 2004). La carta de consentimiento informó a los participantes sobre cualquier riesgo o beneficio relacionado con la participación en el estudio, se les aseguró la confidencialidad a los participantes y la naturaleza voluntaria del estudio.

Por último, la carta de consentimiento proporcionó información de contacto para obtener detalles adicionales sobre el estudio e incluyó la firma tanto para del participante como del investigador (Cone \& Foster, 2004).

Se utilizó un muestreo no probabilístico, de conveniencia. Este tipo de muestreo, permite seleccionar aquellos casos accesibles que acepten ser incluidos. Esto, fundamentado en la conveniente accesibilidad y proximidad de los sujetos para el investigador. Por tanto, se buscó empleados de la industria maquiladora en Ciudad Juárez que manifestaran que en las empresas que trabajaban enfrentaban procesos de cambio organizacional críticos.

La muestra consistió en ocho miembros de cada una de las tres generaciones en estudio Baby Boomers, Generación X y Generación Y. La muestra había enfrentado varias experiencias de cambio, incluyendo reducciones de personal, reestructuraciones, reorganizaciones, fusiones e innovaciones tecnológicas. Finalmente, este investigador trabajó 23 años en la industria maquiladora en niveles de supervisión y gerenciales y tiene acceso a esta población.

\section{Resultados}

El estudio recogió durante el periodo de enero a mayo del 2019, la información de 24 trabajadores de la industria maquiladora en Ciudad Juárez, Chihuahua, México. 
Los datos de la entrevista en el estudio actual se analizaron utilizando el método fenomenológico diseñado por Van Kaam y modificado por Moustakas (1994).

Este método utiliza los siguientes pasos 1. Horizontalización, 2. Reducción y eliminación, 3. Agrupación y tematización de los componentes invariantes, 4. Validación, 5. Descripciones texturales individuales, 6 . Descripciones estructurales individuales y descripciones estructurales texturales de los significados, y 7. Descripción del grupo.

En general, surgieron cinco temas centrales. Las descripciones compuestas proporcionan significado a los temas (Moustakas, 1994). Estos temas ocurrieron a través de las generaciones y a través de diferentes preguntas. Estos temas incluyeron: (a) comunicación (b) participación de los empleados, (c) comprender cómo el cambio impacta a los empleados, (d) percepciones de cambio y (e) percepciones de generaciones.

En cuanto a la comunicación, el $80 \%$ de las respuestas, 20 de 24 participantes, respaldaron el tema de que las comunicaciones son fundamentales para la implementación exitosa del cambio. Los participantes $(60 \%)$ declararon que los mejores métodos de comunicación dependen de la situación. Solo $25 \%$ de los participantes mostraron preferencia por la comunicación cara a cara. Dos participantes, uno de la Generación X y uno de la Generación $\mathrm{Y}$ declararon que las comunicaciones electrónicas eran la mejor manera de comunicar un cambio organizacional importante.

Respecto al involucramiento de los empleados, 19 de 24 participantes respaldaron el tema en la iniciativa de cambio. Este tema surgió a lo largo del estudio y entre generaciones. Adicionalmente, 13 de los 24 participantes declararon que la participación de los empleados era la forma de aumentar la aceptación del cambio por parte de los empleados. Tres participantes declararon que la participación de los empleados era la mejor manera para que los líderes guiaran a los empleados a través de iniciativas de cambio.

En lo que se refiere a la comprensión, 18 de 24 participantes apoyaron el tema de comprender el cambio y cómo el cambio impacta a los empleados.
Cinco participantes declararon que comprender cómo el cambio impacta a los empleados era la mejor manera de guiar a los líderes. En cuanto a la percepción al cambio, Los miembros de todas las generaciones en este estudio manifestaron haber experimentado altas tasas de cambio organizacional. Los 24 participantes habían experimentado una variedad de cambios organizacionales importantes que iban desde reorganizaciones, fusiones, adquisiciones, tecnología, cambios de liderazgo, hasta experimentar el impacto de la globalización.

Los participantes $(50 \%)$ declararon que los cambios habían hecho que el trabajo fuera más exigente o desafiante. Otros participantes (15\%) declararon que los cambios organizacionales habían aumentado su desempeño. Algunos participantes (20\%) declararon que no experimentaron ningún cambio en su desempeño; mientras que otros participantes $(15 \%)$ declararon que los cambios organizacionales habían contribuido a su nivel de frustración.

Finalmente, la percepción entre generaciones arrojó que los 24 participantes respaldaron el tema de las percepciones generacionales. Se percibió que a los baby boomers les desagradaba o se resistían al cambio, se percibió que la Generación X acepta el cambio y se percibe que la Generación Y es más adaptable al cambio y se cuida a sí misma. Los baby boomers establecieron que la Generación X dejaría una organización si no les gustaba el cambio. También declaran que la Generación Y se queja mucho. La Generación X declaró que los baby boomers hace muchas preguntas. La Generación X declaró que a la Generación Y le gusta dar sus opiniones.

\section{Conclusiones}

La industria maquiladora en Ciudad Juárez posee una prestigiada historia y una cultura organizacional fuerte y competitiva, orgullosa de los resultados generados a través de 56 años de inicio del programa maquilador. Historicamente, la industria maquiladora de Ciudad Juárez se ha manifestado por su alta competitividad de su mano de obra calificada y sus innovaciones tecnológicas. 
Este estudio se centró en tres de las cuatro generaciones trabajando en la industria maquiladora de Ciudad Juárez, ya que comprenden la mayor parte de la fuerza laboral (Tulgan, 2004). El estudio actual incluyó tres grupos focales para capturar la experiencia vivida de 24 empleados en diferentes empresas. Todos los participantes habían experimentado cambios organizacionales importantes. La experiencia del participante pareció respaldar el estudio realizado por Johnson-Cramer, Parise y Cross (2007) que reveló que el $85 \%$ de las organizaciones encuestadas habían experimentado iniciativas de cambio significativas en los últimos dos años.

El alcance de este estudio fenomenológico, que incluyó a 24 participantes, fue limitado en el tamaño relativo de la muestra. La generalización no es la intención de la investigación cualitativa; en cambio, la intención es explorar completamente un fenómeno central (Creswell, 2005).

Cinco temas centrales surgieron del estudio actual. Estos temas incluyeron: (a) comunicación, (b) participación de los empleados, (c) comprender cómo el cambio impacta a los empleados, (d) percepciones de cambio y (e) percepciones generacionales.

La necesidad de una comunicación efectiva y abierta para lograr un cambio organizacional exitoso apareció en todos los grupos focales. Estos hallazgos respaldan la literatura que recomienda que los líderes comuniquen con precisión qué cambios se requieren y por qué (Goldberg, 2005). La comunicación ineficaz o la subestimación de los requisitos de capacitación es a menudo la razón por la cual las iniciativas de cambio fallan (Price y Chahal). Lewis sugirió que la comunicación de los empleados juega un papel clave en las iniciativas de cambio (2006).

Las iniciativas de cambio a menudo requieren que los trabajadores aprendan nuevas habilidades; los líderes deben entender que los empleados a menudo se ponen ansiosos cuando se les pide que hagan más o que hagan cambios en su forma de trabajar (Brown, 2003). La mayoría de los participantes declararon que su primera respuesta al cambio organizacional fue hacer preguntas o tratar de comprender el cambio.
Los participantes de las tres generaciones consideraron que los líderes son responsables de garantizar que los empleados comprenden el cambio. Esta idea es apoyada por la literatura que reveló que durante una iniciativa de cambio, los líderes deben ayudar a los empleados a comprender el cambio. Los líderes deben practicar comunicaciones bidireccionales. Los líderes efectivos son conscientes respecto a la importancia de escuchar a los empleados durante una iniciativa de cambio, puesto que es un paso importante para ganarse el respeto, la confianza y el apoyo de los empleados.

Los miembros de todas las generaciones en el estudio actual han experimentado altas tasas de cambio organizacional. En general, los participantes han experimentado una variedad de cambios organizacionales importantes que van desde reorganizaciones, fusiones, adquisiciones, tecnología, cambios de liderazgo, hasta experimentar el impacto de la globalización. El cambio y la incertidumbre se han vuelto comunes en el entorno empresarial; Se debe considerar que tratar los efectos del cambio en todos los aspectos de la organización lleva a una iniciativa exitosa de cambio de gestión (Bunker, 2006).

En general, los participantes percibieron que el cambio organizacional aumentó su desempeño o hizo que su trabajo fuera más exigente, desafiante o frustrante. Aún otros percibieron que los cambios organizacionales no han causado cambios en el desempeño personal. Los participantes creen que la mejor manera de manejar el cambio es abrazar, disfrutar o esperar el cambio. El $45 \%$ de los encuestados declararon que aceptaron, disfrutaron o esperaban cambiar. Estos hallazgos respaldan la literatura que describe el éxito organizacional y personal que depende de la capacidad de aceptar el cambio.

Las respuestas de los 24 participantes respaldaron el tema de las percepciones generacionales. Seung-Bum y Guy sugirieron que las personas perciben y entienden su entorno, especialmente su entorno de trabajo, basándose en circunstancias y eventos educativos, sociales e históricos presentes durante sus años de formación (2006). Los miembros de las cohortes generacionales tienen opiniones definidas sobre cómo los miembros de diferentes generaciones responden a los cambios en el lugar de trabajo. 
La literatura actual muestra una exploración limitada sobre si existen o no diferencias generacionales en respuesta al cambio organizacional. Este estudio aporta a este vacío en el estado del arte.

Los empleados pertenecientes a la generación de los baby boomers fueron percibidos como más resistentes al cambio por las otras generaciones de lo que se creía. Las generaciones $\mathrm{X}$ e $\mathrm{Y}$ describieron a los baby boomers como a los que no les gusta el cambio, que son resistentes al cambio, lentos o que no aceptan el cambio, y se ponen en su camino. Kidwell (2003) sugirió la presencia de estereotipos generacionales y esto puede explicar cómo se perciben los baby boomers. Las respuestas de la Generación X e Y incluyeron el concepto de que los baby boomers son más resistentes y aceptan menos los cambios que las generaciones más jóvenes. Quizás que los baby boomers se opongan a los cambios en las iniciativas que establecieron. Los participantes también indicaron que los baby boomers tienen una idea de cómo deberían ser las cosas y resisten los cambios a esos ideales.

A pesar de que algunos baby boomers describieron a su cohorte como apegados a sus formas, no se percibieron a sí mismos como lentos para cambiarr. Aunque los miembros de la Generación X e Y percibieron que los baby Boomers eran resistentes al cambio y lentos para cambiar, el $50 \%$ de los baby boomers dijeron que realmente disfrutaban o esperaban el cambio. Un número igual de Generación X e Y dijeron que disfrutaron o esperaban cambiar.

Esto no debe sorprender, dada la importancia que muchos entrevistados le otorgaron a la ética de trabajo y las historias sobre su aprendizaje y el énfasis en experiencias organizacionales enfocadas en resultados previos. La teoría de atracción-selecciónabandono (Schneider, 1987) apoyaría la idea de que estos individuos se sintieron atraídos inicialmente por la industria maquiladora en Ciudad Juárez, porque la cultura orientada a resultados se ajustaba a sus preferencias personales.

En general, la Generación X se describió como aceptable o adaptable al cambio. Esta percepción de aceptar y adaptarse al cambio era congruente con la autopercepción de la Generación X.
Los miembros de la generación $X$ se autodescriben como mejores manejando el cambio. Los comentarios de los baby boomers incluyeron el concepto de que era más probable que la Generación X quisiera cambios y probara nuevas formas de hacer las cosas.

Otros participantes comentaron que la Generación X y la Generación Y son similares y ambas generaciones fueron más aceptables de los cambios organizacionales, en relación a los baby boomers; sin embargo, las generaciones $\mathrm{X}$ e Y tienen menos experiencia en los negocios y, de esta manera, son más receptivos a los cambios. Adicionalmente, las generaciones X e Y tienen más probabilidades de rechazar los cambios que afectan su vida personal.

Este concepto está respaldado por la Lancaster y Stillman que revelaron que los miembros de la Generación $\mathrm{X}$ tienen un mayor sentido del equilibrio entre la vida laboral y el trabajo (2002). Otro estudio de Seung-Bum y Guy sugiere que los trabajadores de la Generación $\mathrm{X}$ tienen más probabilidades de cambiar de trabajo que los empleados de las otras generaciones.

Se describió que la generación Y acepta o se adapta al cambio. También se describió que se cuidaban a sí mismos y que probablemente dejarían la organización si no les gustaban los cambios. Estas percepciones se mantuvieron en las tres generaciones.

La literatura refuerza la opinión de que la generación Y es más probable que abandonen una organización si no les gustan los cambios. Según Westerman y Yamamura (2006), la incapacidad de una empresa para cambiar su cultura organizacional y su entorno para satisfacer las necesidades de los empleados de la Generación X e Y dará como resultado la insatisfacción y rotación de los empleados.

Este estudio encontró que la autopercepción de la Generación Y concordaba con las opiniones sostenidas por otras generaciones. Las respuestas de la Generación Y incluyeron la idea de que su generación es algo egoísta y se preocupa de cómo le afectaran los cambios. La Generación Y cree que tiene otras opciones y si no le gustan los cambios, podrían abandonar la organización. 
Los participantes comentaron que la Generación Y ve por sus propios intereses más que otras generaciones. Esperan poder cambiar cuando el cambio proporcione una oportunidad de crecimiento y desarrollo. La generación X considera que la generación Y se absorbió a sí misma y piensa que el mundo gira en torno a ellos. Los participantes se hicieron eco de la opinión de que la Generación Y se apresura para adaptarse al cambio. Se considera que cuando la generación Y acepta un cambio, se encuentra lista para los próximos pasos y se encuentra lista para seguir adelante.

La generación de los baby boomers compartió la opinión de que los miembros de la Generación Y entran en una organización con títulos de posgrado y una mentalidad de hacer cambios y buscar avances personales, independientemente de los costos. La generación Y tiende a no ser leal a la organización y se va en pocos años.

En general los baby boomers consideran que la Generación Y quiere más calidad de vida y equilibrio entre la vida laboral y personal. Los participantes comentaron que la necesidad de calidad de vida significaba que la Generación Y no estaba dispuesta a trabajar más allá del tiempo para dejar de fumar. Los miembros de la Generación Y anhelan el equilibrio de la vida laboral que sus padres no tuvieron.

Una vez que los líderes reconocen estas percepciones, pueden desarrollar planes para reforzar las percepciones positivas y reducir las percepciones negativas. El estudio Kidwell (2003) sugirió la presencia de estereotipos generacionales. Por ejemplo, los trabajadores de más edad estaban interesados en la seguridad laboral y podían resistir los cambios que percibían como riesgosos para su seguridad.

En general, los trabajadores más jóvenes presentaron la percepción de una mayor apertura al cambio y a la oportunidad de participar en los procedimientos de cambio. Estos trabajadores más jóvenes percibieron a los trabajadores mayores como barreras para el éxito (Kidwell, 2003).

Este estudio es importante, la presente investigación ha llevado a cabo una exploración limitada sobre si existen o no diferencias generacionales en respuesta al cambio organizacional.
Este estudio puede ayudar a llenar el vacío existente en el estado de la cuestión actual, respecto a la respuesta de las diferentes generaciones al enfrentar el cambio organizacional. Esta investigación ofrece un panorama no proporcionado anteriormente, destacando las experiencias vividas de los líderes en una muestra representativa de la industria maquiladora en Ciudad Juárez sobre la respuesta de los cohortes generacionales, al cambio organizacional.

La recolección de la información en el estudio actual puede proporcionar a los líderes una idea de cómo adaptar los planes de cambio para cumplir con los requisitos de las diferentes generaciones. Por ejemplo, Los participantes en el estudio actual destacaron la importancia de la participación de los empleados en las estrategias de cambio. Además, de acuerdo con este estudio, los líderes obtienen el conocimiento de las percepciones generacionales y puedne ser capaces de encontrar formas de abordar estas percepciones cuando se trabaja con equipos multigeneracionales.

Este estudio es significativo, los gerentes de las empresas podrían beneficiarse de los resultados obtenidos en el estudio actual. Al tener la oportunidad de personalizar las estrategias de cambio basadas en el comportamiento generacional predictivo, mejorando así su propio éxito como líderes. Los dirigentes en las empresas deben ser conscientes de estas percepciones generacionales y desarrollar mejor las estrategias de cambio y, por lo tanto, mejorar el desempeño organizacional.

Adicionalmente, los resultados del estudio proporcionan información respecto a la comunicación efectiva y su importancia para la implementación exitosa del cambio (Lewis, 2006). Los líderes deben involucrar a los empleados en el proceso de cambio. Los empleados deben comprender el cambio y los líderes deben comprender cómo afecta el cambio a los empleados. Los líderes deben ser conscientes de que existen percepciones de las diferencias generacionales en respuesta al cambio organizacional.

Actualmente, las empresas de todos los sectores tienen el desafío de aumentar su capacidad de cambio, no solo por la competencia y la tecnología, sino también en preparación para esos cambios (Kerber y Buono, 2005). 
Lo que complica aún más la situación es la presencia de tres generaciones activas y una ya de salida en la fuerza laboral. Un estudio de 2006 reveló que el 43\% de las organizaciones encuestadas reconocieron que los cambios demográficos representaban un impacto significativo en sus organizaciones (Lesser y Rivera, 2006).

A pesar de que las empresas están de acuerdo en quee los cambios demográficos son una situación importante en el funcionamiento de las organizaciones, la mayoría aún no sabe cómo adaptarse a la fuerza laboral cambiante (Lesser y Rivera, 2006). Las organizaciones necesitan adaptarse a este cambio demográfico. Basado en los resultados del estudio actual, este estudio proporciona varias recomendaciones para los dirigentes de las empresas.

\section{Estas recomendaciones incluyen el conocimiento delas características generacionales, mejorar las comunicación del cambio organizacional y fortalecer la participación de los empleados. Estas recomendaciones se analizan a continuación, seguidas de recomendaciones para estudios posteriores.}

Según la revisión de la literatura y los resultados del estudio actual, los líderes requieren comprender las características de las cohortes generacionales. Los líderes deben ser conscientes de que los teóricos reconocen que no todos los miembros de una generación tienen creencias o comportamientos idénticos (Strauss y Howe, 1992). Los participantes en el estudio actual sostuvieron percepciones positivas $y$ negativas de cómo los miembros de diferentes generaciones responden al cambio organizacional.

Los líderes deben ser conscientes de estas percepciones y encontrar formas de abordarlas cuando trabajen con equipos multigeneracionales. Los trabajadores más jóvenes son percibidos como un cambio acogedor. Los trabajadores más jóvenes perciben a los trabajadores mayores como barreras para el éxito.

El estudio posee varias fortalezas. Una de ellas es el análisis dentro de la industria maquiladora. El enfoque cualitativo utilizado, permite una exploración diferente a los realizados en otros estudios.
Las entrevistas realizadas y los hallazgos sobre el cambio organizacional y su impacto en los diferentes cohortes generacionales son enriquecedores a la teoría administrativa.

Hay una serie de áreas dentro de este tema y en la industria maquiladora que justifican la consideración de futuras investigaciones. Dada la naturaleza de este estudio, un estudio longitudinal adicional con los participantes en este estudio actual podría llevarse a cabo. Una investigación a lo largo del tiempo con este grupo podría revelar indicios de la efectividad del proceso de cambio liderazgo en las empresas maquiladoras.

En particular, los estudios futuros deberían concentrarse en aquellas personas involucradas en el proceso de desarrollo al principio de sus carreras para identificar la efectividad de un cambio a largo plazo y duradero.

Este estudio tambien posee limitaciones. Como se trata de un estudio de método cualitativo, la informacion recolectada de los participantes en la investigación se encuentra relacionada con sus sentimientos, percepciones y experiencias en el contexto de la industria maquiladora en Ciudad Juárez.

\section{Referencias}

Appelbaum, S., Serena, M. y Shapiro, B. (2004). Generation $\mathrm{X}$ and the Boomers: Organizational myths and literary realities. Management Research News, 27(11/12), 1-28.

Arsenault, P. (2004). Validating generational differences: A legitimate diversity and leadership issue. Leadership \& Organization Development Journal, 25(2), 124-141.

Axelrod, R. H., Axelrod, E., Jacobs, R. W., \& Beedon, J. (2006). Beat the odds and succeed in organizational change. Consulting Management-C2M, 17(2), 6-9.

Bauman, Z. (2003). Intimations of postmodernity. Routledge.

Bauman, Z. (2001). Modernidad líquida. Buenos Aires. Fondo de Cultura Económica.

Brown, D. C. (2003). Leading complex change. Behavioral Health Management, 23(6), 25-27. 
Bustillos, S., Rincones, R., Jiménez, P. y Hernández, J. (2009). Los otros nómadas: movilidad laboral internacional de personas altamente calificadas. Universidad Autónoma de Ciudad Juárez.

Coleman, A. (2006). How to embrace change. Director, 60(5), 33.

Cone, J. D., \& Foster, S. L. (2004). Dissertations and theses from start to finish. Washington, DC: American Psychological Association.

Contreras, Óscar. 2000. Empresas globales, actores locales. Producción flexible y aprendizaje industrial en las maquiladoras. México: El Colegio de México.

Contreras, O. y Munguía, L. (2007). Evolución de las maquiladoras en México. Política industrial y aprendizaje tecnológico. Región y Sociedad. Volumen XIX. Número especial, México.

Creswell, J. W. (2005). Educational research: Planning, conducting, and evaluating quantitative and qualitative research (2nd ed.). Upper Saddle River, NJ: Pearson.

Creswell, J. W. (2003). Research design: Qualitative, quantitative, and mixed methods approaches (2nd ed.). Thousand Oaks, CA: Sage Publications.

Creswell, J. (1998), "Qualitative inquire and research", Thousand Oaks, CA: Sage Publications.

Crow, G. Wiles, R. Heath, S., \& Charles, V. (2006). Research ethics and data quality: The implications of informed consent. International Journal of Social Research Methodology, 9(2), 83-95.

Denzin, N. y Lincoln, Y. (1998), “Collecting and interpreting qualitative materials", Thousand Oaks, CA: Sage Publications.

Earle, H. (2003). Building a workplace of choice: Using the work environment to attract and retain top talent. Journal of Facilities Management 2 (3): 244-257.

Eisner, S. (2005). Managing generation Y. Engineering Management Review 39 (2): 6-18.
Ferri-Reed, J. (2010). The keys to engaging Millennials. Journal for Quality and Participation, 33(1), 31.

Glass, A. (2007). Understanding generational differences for competitive success. Industrial and Commercial Training, 39(2), 98-103.

Gonzalez, C. (2006). A causal comparative study of work ethic as a function of generational cohort. (Doctoral dissertation, University of Phoenix, 2006).

Harris, P. (2005). Boomer vs. Echo Boomer: The work war? Training and Development 59 (5): 44-49.

Hernández, J. (2018). Desarrollo organizacional: Teoría, prácticas y casos. México. Pearson Educación.

Hernández, J., Espinoza, J., y Aguilar Arellano, M. (2015). Diferencias en los motivadores y los valores en el trabajo de empleados en empresas maquiladoras. Contaduría y administración, 61(1), 58-83.

Higgs, M., \& Rowland, D. (2005). All changes great and small: Exploring approaches to change and its leadership. Journal of Change Management, 5(2), 121-151.

Hoover, E. (2009). The Millennial muddle. Chronicle of Higher Education, 56(8), 1-34.

Howe, N. y Strauss, W. (2000). Millennials Rising: The next great generation. New York, NY: Vintage.

Jefferies, F. \& Hunte, T. (2003). Generations and motivation: A connection worth making. Journal of Behavioral and Applied Management, 6(1), 37-70.

Johnson-Cramer, M., Parise, S., \& Cross, R. L. (2007). Managing change through networks and values. California Management Review, 49(3), 85-109.

Kerber, K., \& Buono, A. F. (2005). Rethinking organizational change: Reframing the challenge of change management. Organizational Development Journal, 23(3), 23-38. 
Kidwell, R. (2003). Helping older workers cope with continuous quality improvement. Journal of Management Development, 22(10), 890-905.

Kotter, J. P. (2007). Leading change. Harvard Business Review, 85(1), 96-103.

Lancaster, L. C., \& Stillman, D. (2002). When generations collide: Who they are, why they clash, how to survive the generational puzzle at work. New York: Harper Business.

Lesser, E., \& Rivera, R. (2006). Closing the generational divide. IBM Global Business Services.

Lewis, L. K. (2006). Employee perspective on implementation communication as predictors of perceptions of success. Western Journal of Communications, 70(1), 23-46.

Lipovestky, G. (1990). El imperio de lo efímero. Barcelona. Ed. Anagrama.

MacDonald, B. H. (2007). When numbers alone are not enough: Applications of qualitative methodologies. Feliciter, 53(1), 26-29.

Mannheim, K. (2013). Essays on the sociology of knowledge. New York: Oxford University Press.

Markert, J. (2004). Demographics of age: Generational and cohort confusion. Journal of Current Issues and Research in Advertising, 26(2), 11-25.

McAfee, A. (2006). Mastering the three worlds of information technology. Harvard Business Review, 84(11), 141-149.

Mckenzie, D. y Sasin, M. (2007). Migration, Remittances, Poverty and Human Capital. Conceptual and Empirical Changes. World Bank Policy Research Working Paper. Washington.

McNamara, S. (2005). Incorporating generational diversity. Association of Operating Room Nurses. AORN Journal, 81, 1149-1153.

Morton, L. (2001). Segmenting publics: Segmenting Baby Boomers. Public Relations Quarterly 46(3): 46-47.

Moustakas, C. (1994). Phenomenological research methods. Thousand Oaks, CA: Sage Publications.
Murphy, M. (2005). Why CEOs get fired. Leadership Excellence, 22(9), 13.

Neuman, W. L. (2003). Social research methods: Qualitative and quantitative approaches (5th ed.). NY: Prentice Hall.

Patton, M. Q. (2002). Qualitative research and evaluation methods (3rd ed.). Thousand Oaks, CA: Sage Publications.

Peiró, J. (2004). El sistema de trabajo y sus implicaciones para la prevención de los riesgos psicosociales en el trabajo. Univ. Psychol. Bogotá, Colombia. 3 (2): 179-186, juliodiciembre.

Price, A. D. F., \& Chahal, K. (2006). A strategic framework for change management. Construction Management and Economics, 24, 237-251.

Rainer, T. y Rainer, J. (2011). The Millennials: Connecting to America's largest generation. Nashville, TN: R \& H Publishing.

Rawlins, C., Indvik, J. y Johnson, P. (2008). Understanding the new generation: What the Millennial cohort absolutely, positively must have at work. Journal of Organizational Culture, Communications, \& Conflict, 12(2), 1-8.

Reynolds, C. (2004). Boomers, act II. American Demographics 26 (8): 10-11.

Santos, B. (2006). Renovar la teoría crítica y reinventar la emancipación social (encuentros en Buenos aires). Clacso Libros.

Schlesinger, A. (1999). The cycles of American history. Boston: Houghton Mifflin Harcourt.

Schneider, B. (1987). The people make the place. Personnel psychology, 40(3), 437-453.

Seung-Bum, Y., \& Guy, M.E. (2006). GenXers versus Boomers work motivators and management implementations. Public Performance and Management Review, 29(3), 267-284.

Simons, N. (2010). Leveraging generational work styles to meet business objectives. Information Management, 44(1), 28-33.

HERNÁNDEZ-PALOMINO, Jorge Arturo. Análisis cualitativo de la reacción al cambio de tres generaciones de empleados en la industria maquiladora. Revista Transdisciplinaria de Estudios Migratorios. 2019 
Strauss, W., \& Howe, N. (1992). Generations: The History of America's Future, 1584 to 2069. N Y: William Morrow \& Co.

Sturges, J. y Guest, D. (2004). Working to live or living to work? Work/life balance early in the career. Human Resource Management Journal 14 (4): 5-20.

Sujansky, J. (2004). Leading a multigenerational workforce. Occupational health \& safety 73 (4): 16-18.

Swenson, C. (2008). Next generation workforce. Nursing Economics, 26(1), 64-60.

Tolbize, A. (2008). Generational differences in the workplace. Research and training center of community living, 19, 1-13.

Tulgan, B. (2004). Trends point to a dynamic generational shift in the future workforce. Employment Relations Today, 23-31.

Twenge, J., Konrath, S., Foster, J., Campbell, K., y Bushman, B. (2008). Egos inflating over time: A cross-temporal meta-analysis of the Narcissistic Personality Inventory. Journal of Personality, 76(4). 875-902.

Westerman, J. W., \& Yamamura, J. H. (2006). Generational preferences for work environment fit: Effects on employee outcomes. Career Development Journal, 12(2), 150-161.

Weston, M. (2006). Integrating generational perspectives in nursing. Online Journal of Issues in Nursing, 11(2), 12.

White, W. (2006). Four generations learning to work better together. Public Management, 35.

Williams, A. y Nussbaum, J. (2001). Intergenerational communication across the life span. Mahwah, NJ: Lawrence Erlbaum Associates.

Zemke, R. (2001). Here Come the Millennials. Training, 38 (7): 44. 